\title{
Chitosan Applications Used in Medical Therapy of Tissue Regeneration
}

\author{
Alef Mustafa \\ UMF "Carol Davila" Bucharest, Faculty of Pharmacy, Bucharest, Romania
}

Ana-Maria Ionescu

"Ovidius" University of Constanta, Faculty of Medicine, Department IV

Clinical Disciplines, Constanta, Romania

Melat Cherim

UMF Carol Davila Bucharest, Faculty of Pharmacy, Bucharest, Romania

Rodica Sîrbu

"Ovidius" University Of Constanţa, Faculty Of Pharmacy, Constanta, Romania

\begin{abstract}
Chitosan is an unique natural biopolymer that has great potential in tissue engineering applications and over the past several decades, it has emerged as a promising biomaterial for biomedical applications. Due to its various properties such as controllable biodegradability, biocompatibility, antimicrobial activity and functionalizability, chitosan can be used to form chitosan-based scaffolds and in different scaffold fabrication techniques. Over the years a great number of studies have been performed to evaluate the cytocompatibility of chitosan using a variety off cell types such as osteoblasts, chondrocytes, fibroblasts, nucleus pulposus cells, neutral and endothelial cells. It was shown that chitosan is biocompatible with these cell types and has the potential to be used for bone, cartilage, skin, intervertebral disc, ligament and tendon, and nerve and vascular tissue engineering. The flexibility of the processing conditions of chitosan aids in the fabrication of versatile substrates as scaffolds for tissue regeneration or carriers for biological molecules. It is critical to synthesize medical grade chitosan materials with controllable structure and properties that will allow the development of chitosan-based medical devices and it is beneficial to chemically design chitosan derivatives with molecular and biological specificity through bulk material modification. Despite all the challenges, chitosan holds great promise as a biomaterial for developing medical products and medical therapies.
\end{abstract}

Keywords: natural biopolymer, chitosan, tissue regeneration, biomedical applications

\section{Introduction}

Over the past years, the medical and pharmaceutical fields faced with an increasing number of patients suffering from wounds and burns difficult to treat and heal. The dressing is important during the wound healing process because it protects the injury and contributes to the recovery of dermal and epidermal tissues. Some natural polymers such as polysaccharides (alginates, chitin, chitosan, heparin, chondroitin), proteoglycans and proteins (collagen, gelatin, fibrin, keratin, silk fibroin, eggshell membrane) are extensively used in wounds and burns management, due to their biocompatibility, biodegradability and similarity to macromolecules recognized by the human body. During the wound healing process, dressings are used for the regeneration and repairing of dermal and epidermal tissues. Wound dressing materials protect the wound mainly against microorganisms acting such as physical barriers permeable for moisture and oxygen [1]. For maintaining an optimally moisture and for the stimulation of wound healing is essential to apply a passive dressing. Due to their influence 
on local cellular response, several products such as hydrogels, foams, hydrocolloids (carboxymethyl-cellulose), alginate, collagen, cellulose, cotton/rayon, transparent films (polyurethane) are recommended as passive dressings for wounds and burns. [2][3] These products have some very useful properties, and among them the most important are the following: protection of the periwounded skin, maintaining a suitable moisture at the wound level, preventing and keeping under control microbial biofilms, cleansing the injured tissues, eliminating/minimizing pain, removing dead spaces and nonviable tissues, controling the odors. [4][5][6] In order to improve the wound healing process the natural biopolymers can be mixed along side with some natural products having emollient, demulcent, epithelializing, astringent, antimicrobial, antiinflammatory and antioxidant properties. [7] Active wound dressings are impregnated with antimicrobials (topical antibiotic and antifungal products), collagen or enzyme debriding agents.

In the case of chronic wounds in order to prevent a local infection, antimicrobials are commonly used. Some examples of such antimicrobial substances are: silver sulfadiazine, methylene blue, crystal violet, honey, polyhexamethylene biguanide (PHMB) and cadexomeriodine. [8] There are certain factors that influence the frequency of dressing change, such as the presence of infection, the accumulation of fluid and debris, the cleanliness and integrity of bandage.[9]

Natural polymers such as collagen, chitosan, elastin, fibrinogen are biocompatible substrates similar to macromolecules recognized by the human body. They are used in regenerative medicine for wounds and burns dressing because of their biocompatibility, biodegradability and similarity to the ECM and for human epithelial stem cells culture or in vitro reconstituted epithelia. [10,11]

In the last decade, chitosan has been extensively used in biomedical applications. In wound healing, a great amount of research has investigated the use of different chitosan formulations for skin wound closure in animal models. [12,13] Chitosan is the active component of a number of wound-dressing products on the United States market such as Tegasorb $^{\mathrm{TM}}$, Chito-Seal ${ }^{\circledR}, \mathrm{HemCon}^{\mathrm{TM}}$, and Syvek Patch $\circledast[14]$ and used in the battlefield attributing to the hemostatic and antimicrobial properties of chitosan. Since the late 1980s, there has been an explosion of research on evaluating a broad range of polymeric, ceramic, and metallic biomaterials for regenerating tissues. Chitosan, a naturally occurring biopolymer, has attracted much research interest in tissue engineering applications because of its biocompatibility, biodegradability, antimicrobial properties, and functionality. [15] Inducing and stimulating the wound healing process, natural polymers are involved in the repair of damaged tissues and consequently in skin regeneration. [16] Due to their threedimensional crosslinked polymeric networks that are soaked with water or biological fluids, biomaterial hydrogels are employed in the pharmaceutical and biomedical area, especially for wound management and tissue engineering. [17]

\section{Research Methods}

Chitin is the most abundant natural amino polysaccharide (poly-N-acetyl-glucosamine) produced annually almost as much as cellulose. It is the major component of the invertebrates, crustaceans and insects exoskeleton and of the fungi cell wall. $[18,19,20]$ Structurally, chitosan is a poly-N-acetyl-glucosaminoglycan made of randomly distributed $-(1 \rightarrow 4)-\mathrm{d}$ glucosamine and $\mathrm{N}$-acetyl-d-glucosamine. [21] Chitosan is obtained by alkaline deacetylation of chitin, respectively by treating shrimp and other crustacean shells with sodium hydroxide. [22] One of the most intriguing and important features of chitosan is its ability to form hydrogels at mild conditions. These hydrogels are suitable to be administered in a minimally invasive manner. Various chitosan-based hydrogel systems that respond to stimuli such as light, $\mathrm{pH}$, ionic concentration, chemical and physical reactions, and temperature have been developed as injectable systems for regenerative applications. For example, by introducing photosensitive moieties (lactose and azide) onto the chitosan backbone and then by photo-cross-linking, we could obtain photo-cross-linkable chitosan systems. [23] It is a fact thet thermosensitive chitosan hydrogels are among the most extensively investigated in situ gelling systems.

\section{Scaffold Fabrication Techniques}

Acording to Laurencin et al. tissue engineering is defined as: "the application of biological, chemical, and engineering principles to the repair, restoration, or regeneration of living tissues by using biomaterials, cells, and factors alone or in combination" [24] Scaffold-based tissue engineering is one of the most well-studied approaches to regenerating different types of tissues, which involves seeding cells together with certain signaling molecules on a three-dimensional (3D) porous biodegradable matrix, culturing them in vitro and implanting them into in vivo defects. In this therapeutic approach, biomaterials, usually in the form of 3D porous scaffolds, play multiple significant roles to provide structural maintenance of 
the defect shape, void volume for vascularization, and serve as temporary extracellular matrix (ECM) for cell adhesion, proliferation, differentiation, and maturation. In addition, scaffolds can act as a delivery vehicle for bioactive molecules, growth factors, and cells to the defect site for tissue morphogenesis and defect healing. [25]

Attributing to the ease of processing of chitosan and using different techniques a variety of 3D chitosan scaffolds have been fabricated, was One of the first recorded techniques used to prepare porous scaffolds was solvent casting, in combination with particulate leaching. [26,27] In this technique the process involves first dissolving a polymer in an organic solvent. This formed polymer solution is then mixed with particles, such as salt and sugar, particles that present specific dimensions . The mixture of polymer/particles/ solvent is shaped into its final geometry by casting in a predefined mold and allowing the solvent to evaporate. Following the solvent evaporation, the composite material formed from polymer and particles is then immersed in a suitable solvent to dissolve the particles, leaving behind a porous structure. In one attempt, Lim et al. prepared porous chitosan scaffold from a mixture of acidic chitosan solution and sodium acetate particles as the porogen. [28] It was noticed that if the ratio of sodium acetate increased it led to the increase of the porosity and interconnectivity in consequence. As a final result it was noticed that between the main pores (200-500 $\mu \mathrm{m})$ were present many minute pores $(7-30 \mu \mathrm{m})$ for the scaffold prepared with $90 \%$ sodium acetate. Althogh the process in very simple and versatile, this technique is limited by the lack of control over the scaffold interconnectivity. Residual porogen particles are potentially associated with the resultant structures and also the mechanical properties of the scaffolds prepared by this method are relatively low.

Another widely used method for the preparation of porous scaffolds is thermally induced phase separation. [29] This process consists in the phase separation of the homogeneous polymer solution by cooling the solution below the solvent melting temperature. This leads to solid-liquid demixing and results in the formation of two frozen phases: a phase poor in polymer and a phase rich in polymer. Subsequent removal of the solvent crystals in the demixed solution through vacuum sublimation leaves a porous polymer scaffold. [30] Madihally et al. were among the first to investigate the feasibility of fabricating highly porous 3D chitosan scaffolds with different architectures by using this method. [31] It is possible to obtain mean pore diameters of 1-250 $\mu \mathrm{m}$ by varying the freezing conditions. It was demonstrated thet this process generally produces highly interconnected porous scaffolds with porosities up to $95 \%$. Also using this method, in addition to pure chitosan scaffolds, porous chitosan/ceramic composite scaffolds have been fabricated. [32]

By treating aqueous acidic solution of chitosan with glycerophosphate salts or inorganic phosphate salts the chitosan gel formed a neutral physiological temperature setting. This is another interesting example of thermogelling system. The gelation has been attributed to a combination of favorable properties such as hydrogen bonding, electrostatic interactions, and hydrophobic interactions between polymer chains and glycerophosphate salts. The glycerophosphate salts are represented by polyols bearing single anionic groups. [33]

Due to its great biological and chemical properties such as biodegradability, biocompatibility, and especially the antibacterial and antimicrobial activity, chitosan and its derivatives have been widely investigated as wound-dressing materials and as scaffolds for skin tissue engineering. In order to treat severe burn wounds antibiotic-loaded chitosanbased scaffolds have been used. As an example, there are studies upon artificial skin substitutes made of chitosan membranes or porous scaffolds that have the ability to release antibacterial agents such as nanotitanium dioxide or silver sulfadiazine [34] and exhibit good antimicrobial properties. For preventing the wound from infections, which represent a major problem in the regeneration process of the injuty, chitosan-based scaffolds can play more important roles such as carriers for growth factors to accelerate skin wound healing. A group of researchers, Obara et al. [35,36] prepared a photocross-linkable chitosan. In order to produce hydrogels the chitosan molecules were modified by lactose and photoreactive azide and they were photo-cross-linked under brief UV irradiation. The fibroblast growth factor-2 (FGF-2) was dispersed into the aqueous azide-chitosan-lactose solution and photo-cross-linked to form a hydrogel, leading to accelerated wound closure (Figure1.a and d). 

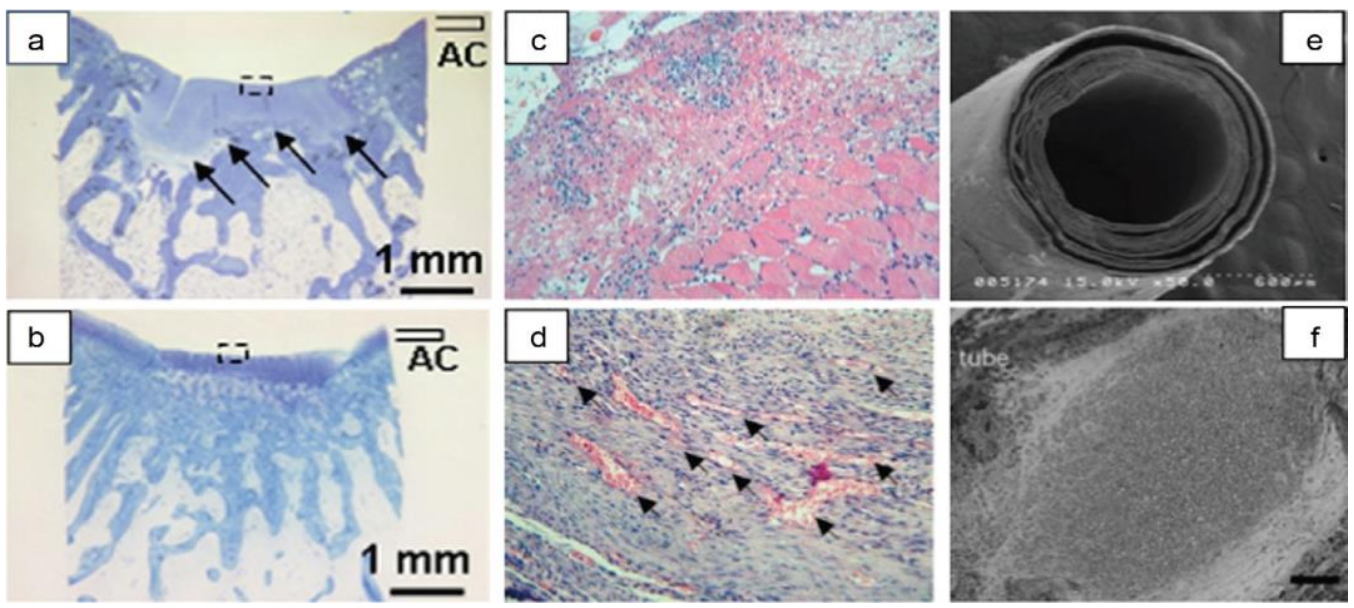

FIGURE 1. Porous chitosan scaffolds for the regeneration of soft tissues. (a) and (b), (c) and (d): Chitosan hydrogel for the repair of skin. (e) and (f): Chitosan nanofibrous tubes (e) to repair a sciatic nerve defect. Regenerated nerve tissue formed within the inner space of the tube and the sprouting of myelinated axons (f).[37]

\section{Results and Discussions}

Chitosan is a unique natural polymer that has great potential in tissue engineering applications. This is due to its controllable biodegradability, biocompatibility, antimicrobial activity, and functionalizability. There have been numerous studies and research to evaluate the cytocompatibility of chitosan. Different cell types such as osteoblasts, chondrocytes, fibroblasts and endothelial cells have been used for these studies and the results have shown that chitosan has the propertie of nontoxicity and can support these cell types to adhere and proliferate. This result can suggest that chitosan is biocompatible with these cell types and has the potential to be utilized for skin and tissue regeneration.

For the local hemostatic antibacterial, anti-inflammatory and wound healing properties, different formulations of chitin and chitosan were obtained:

- $\quad$ water-soluble chitin (WSC) ointment as a wound heal-ing assistant,

- microcrystalline partially deacetylatedchitin hydrochloride as promising hemostatic material,

- bactericidal films based on chitin and silver nanopar-ticles for wound dressing applications,

- chitin-based wound dressing containing silver sulfadiazine,

- chitin hydrogel-nano zinc oxide composite bandage,

- phosphorylated chitinand chitosan,

- bioactive chitin/chitosanhydrogel membranes and scaffolds cocultured with keratinocyteand fibroblast cells,

- $\quad$ films, sponges and hydrogels of microcrystalline chitosan withantimicrobial and wound-healing effects for wounds and burns,

- chitosan mesh membrane with a $75 \%$ degree of deacetylation as a wound-healingdressing,

- antibacterial chitosan-nanosilver film,

- chitosan film enriched with an antioxi-dant agent, taurine, in fenestration defects,

- chitosan film/gel containing fucoidan as a wound dressing fordermal burn healing,

- chitosan-Aloevera membranes for wound dressing,

- chitosan films incorporated with thyme oil forpotential wound healing applications,

- chitosan/tricalcium phosphate sponges as tissue engineering scaffoldsfor bone regeneration,

- chitosan-cellulose-silvernanoparticle composite films,

- chitosan-alginate polyelectrolyte complex membranes for wounddressing applications,

- chitosan-gelatin sponge-like/composite films for wound-healing dressing, 
- ciprofloxacin loaded chitosan-gelatin composite films,

- chitosan gel formulation containing epidermal growth factor (EGF) for burn wound healing,

- graft copolymerized chitosan by choosing various types of side chains for drug delivery and tissue engineering,

- novel chitosan-Ca3V10028complex mem-brane with sustained antimicrobial capability for wound dressing,

- hydrophilic, biocompatible and biodegradableTencel-chitosan-pectin composite,

- chitosan-silkfibroin composite nanofibers for wound-dressing applications,

- chitosan-fibrin microbeads for cell delivery in tissue repair applications,

- chitosan-fibrin-sodium alginate composite for wound dressing,

- microporous chitosan hydrogel-nanofibrin composite bandage for skin tissue regeneration. [38]

In order to combine the beneficial features of different components, chitosan-based blend or composite nanofibers have also been fabricated via electrospinning using chitosan and a second polymeric or ceramic component such as poly(lactic

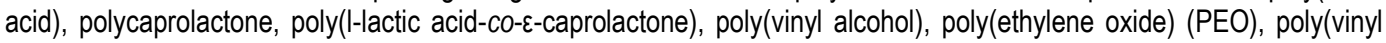
pyrrolidine), poly(ethylene terephthalate), or calcium based ceramics. [37]

A series of various miniaturized chitosan structures fabricated by different techniques is shown in Figure 2 .
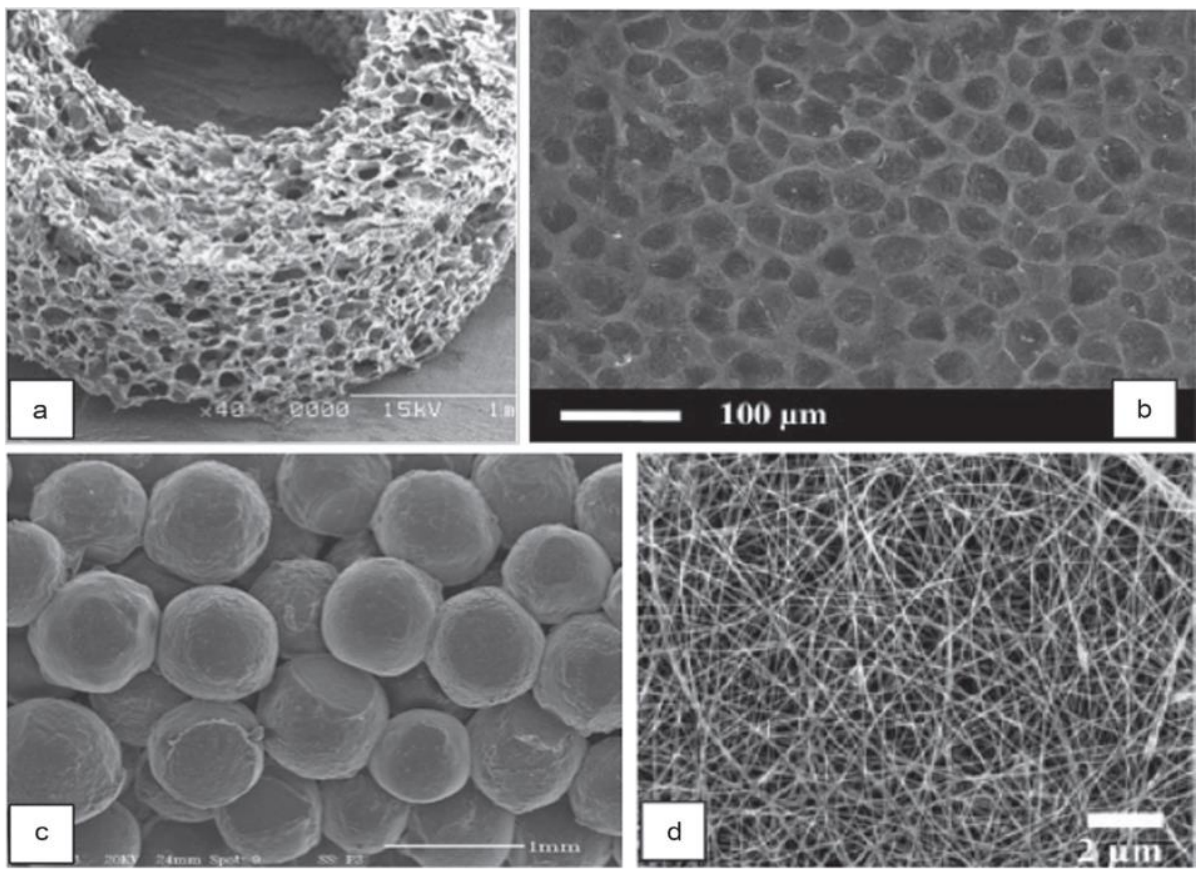

FIGURE 2. Different types of porous chitosan scaffolds fabricated by (a) thermally induced phase separation, (b) dense CO2 gas foaming, (c) sintering chitosan microspheres, and (d) electrospinning. [37]

\section{Conclusion}

In the last years, chitosan received great attention in the research fields of medicine as a promising biomaterial for biomedical applications due to its natural origin and structural similarity to glycosaminoglycans naturally present in native tissues. Furthermore its biological properties such as biocompatibility, biodegradability, antimicrobial activity and functionalizability make it an attractive biomaterial for tissue engineering and regeneration and also drug delivery. 
In the management of wounds and burns dressings play a very significant role. In the process of tissue engineering and regeneration the use of three-dimensional polymeric scaffolds for cell targeting is already a common strategy. The upcoming reseaches and studies of biocompatible and biodegradable natural/synthetic polymers will develop and create new types of wound dressings and lead to innovative applications in regenerative medicine. That is why all the new and recent studies are based on natural polymers such as polysaccharides(chitin, chitosan, alginates, heparin, chondroitin), proteoglycansand proteins (collagen, gelatin, fibrin, keratin, silk fibroin, eggshellmembrane).

\section{References}

[1] [1] Adamian, A.A., Dobysh, S.V., Kilimchuk, L.E., Shandurenko, I.N., Chekmareva, I.A.,2004. Development of new biologically active dressings and methodology oftheir use. Khirurgiia (Mosk) 12, 10-14

[2] [2] Livshits, V.S., 1988. Polymer dressings for wounds and burns (review). Pharm. Chem.J. 22, 515-522

[3] [3] Cornelius, V.J., Majcen, N., Snowden, M.J., Mitchell, J.C., Voncina, B., 2007. Preparationof SMART wound dressings based on colloidal microgels and textile fibres. SPIEInternational Symposium on Smart Materials, Nano- and Micro-Smart Systems,December 11-13, 2006, Adelaide, Australia. Proc. SPIE - Int. Soc. Opt. Eng. $6413,64130 X$

[4] [4] Seaman, S., 2002. Dressing selection in chronic wound management. J. Am. Podiatr.Med. Assoc. 92, 2433.

[5] [5] Boateng, J.S., Matthews, K.H., Stevens, H.N., Eccleston, G.M., 2008. Wound healingdressings and drug delivery systems: a review. J. Pharm. Sci. 97, 2892-2923.Bragulla, H.H., Homberger, D.G., 2009. Structure and functions of keratin proteins insimple, stratified, keratinized and cornified epithelia. J. Anat. 214, 516-559.

[6] [6] Sawant, S.V., Sankpal, S.V., Jadhav, K.R., Kadam, V.J., 2012. Hydrogel as drug deliverysystem. Res. J. Pharm. Technol. 5, 561-569

[7] [7] Mogosanu, G.D., Popescu, F.C., Busuioc, C.J., Pârv anescu, H., Lasčar, I., 2012. Naturalproducts locally modulators of the cellular response: therapeutic perspectivesin skin burns. Rom. J. Morphol. Embryol. 53, 249262

[8] [8] Zilberman, M., Elsner, J.J., 2008. Antibiotic-eluting medical devices for various appli-cations. J. Control Release 130, 202-215

[9] [9] Stojadinovic, A., Carlson, J.W., Schultz, G.S., Davis, T.A., Elster, E.A., 2008. Topicaladvances in wound care. Gynecol. Oncol. 111, S70-S80.

[10] [10] Guerra, L., Dellambra, E., Panacchia, L., Paionni, E., 2009. Tissue engineering for dam-aged surface and lining epithelia: stem cells, current clinical applications, andavailable engineered tissues. Tissue Eng. B Rev. 15, $91-112$

[11] [11] Natesan, S., Zamora, D.O., Suggs, L.J., Christy, R.J., 2012. Engineering a bilayeredhydrogel to control ASC differentiation. J. Vis. Exp. 63, 3953

[12] [12] H. Ueno, H. Yamada, I. Tanaka, N. Kaba, M. Matsuura, M. Okumura, et al., Accelerating effects of chitosan for healing at early phase of experimental open wound in dogs, Biomaterials 20 (1999) 1407-1414

[13] [13] H. Ueno, T. Mori T.Fujinaga,Topical formulations and wound healing applications of chitosan, Adv. Drug Deliv. Rev. 52 (2001)105-115

[14] [14] T.H. Fischer, R. Connolly, H.S. Thatte, S.S. Schwaitzberg, Comparison of structural and hemostatic properties of the poly-N-acetyl glucosamine Syvek Patch with products containing chitosan, Microsc. Res. Technol. 63 (2004) 168-174

[15] [15] E. Khor, L.Y. Lim, Implantable applications of chitin and chitosan, Biomaterials 24 (2003) 2339-2349

[16] [16] Huang, S., Fu, X., 2010. Naturally derived materials-based cell and drug deliverysystems in skin regeneration. J. Control. Rel. 142, 149-159

[17] [17] Das, N., Bera, T., Mukherjee, A., 2012. Biomaterial hydrogels for different biomedicalapplications. Int. J. Pharma Bio Sci. 3, P586-P595

[18] [18] Shahidi, F., Abuzaytoun, R., 2005. Chitin, chitosan, and co-products: chemistry, pro-duction, applications, and health effects. Adv. Food Nutr. Res. 49, 93-135

[19] [19] Jayakumar, R., Reis, R.L., Mano, J.F., 2006. Chemistry and applications of phosphory-lated chitin and chitosan. E-Polymers $35,1-16$

[20] [20] Shi, C., Zhu, Y., Ran, X., Wang, M., Su, Y., Cheng, T., 2006. Therapeutic potential ofchitosan and its derivatives in regenerative medicine. J. Surg. Res. 133, 185-192

[21] [21] Viney, C., Harish, D., 2002. Chitosan - preparation \& properties. Indian Drugs 39,191-194 
[22] [22] Andres, Y., Giraud, L., Gerente, C., Le Cloirec, P., 2007. Antibacterial effects of chitosanpowder: mechanisms of action. Environ. Technol. 28, 1357-1363

[23] [23] M. Ishihara, K. Nakanishi, K. Ono, M. Sato, M. Kikuchi, Y. Saito, et al., Photocrosslinkable chitosan as a dressing for wound occlusion and accelerator in healing process, Biomaterials 23 (2002) 833-840

[24] [24] C.T. Laurencin, A. Ambrosio, M. Borden, J. Cooper Jr., Tissue engineering: orthopedic applications, Annu. Rev. Biomed. Eng. 1 (1999) 19-46

[25] [25] X.H. Liu, P.X. Ma, Polymeric scaffolds for bone tissue engineering, Ann. Biomed. Eng. 32 (2004) 477-486

[26] [26] A.G. Mikos, A.J. Thorsen, L.A. Czerwonka, Y. Bao, R. Langer, D.N. Winslow, et al., Preparation and characterization of poly(l-lactic acid) foams, Polymer 35 (1994) 1068-1077

[27] [27] A.G. Mikos, G. Sarakinos, S.M. Leite, J.P. Vacant, R. Langer, Laminated three-dimensional biodegradable foams for use in tissue engineering, Biomaterials 14 (1993) 323-330.

[28] [28] J. Lim, Y. Lee, J. Shin, K. Lim, Preparation of interconnected porous chitosan scaffolds by sodium acetate particulate leaching, J. Biomater. Sci. Polym. Ed. 22 (2011) 1319-1329

[29] [29] C. Schugens, V. Maquet, C. Grandfils, R. Jerome, P. Teyssie, Polylactide macroporous biodegradable implants for cell transplantation. II. Preparation of polylactide foams by liquid-liquid phase separation, J. Biomed. Mater. Res. 30 (1996) 449-461

[30] [30] F.J. O'Brien, B.A. Harley, I.V. Yannas, L. Gibson, Influence of freezing rate on pore structure in freeze-dried collagen-GAG scaffolds, Biomaterials 25 (2004) 1077-1086

[31] [31] S.V. Madihally, H.W. Matthew, Porous chitosan scaffolds for tissue engineering, Biomaterials 20 (1999) 1133-1142

[32] [32] Y. Zhang, M. Zhang, Synthesis and characterization of macroporous chitosan/calcium phosphate composite scaffolds for tissue engineering, J. Biomed. Mater. Res. 55 (2001) 304-312

[33] [33] C. Hoemann, J. Sun, A. Legare, M. McKee, M. Buschmann, Tissue engineering of cartilage using an injectable and adhesive chitosan-based cell-delivery vehicle, Osteoarthr. Cartil. 13 (2005) 318-329

[34] [34] F.-L. Mi, Y.-B. Wu, S.-S. Shyu, J.-Y. Schoung, Y.-B. Huang, Y.-H. Tsai, et al., Control of wound infections using a bilayer chitosan wound dressing with sustainable antibiotic delivery, J. Biomed. Mater. Res. 59 (2002) 438-449

[35] [35] K. Obara, M. Ishihara, M. Fujita, Y. Kanatani, H. Hattori, T. Matsui, et al., Acceleration of wound healing in healing-impaired db/db mice with a photocrosslinkable chitosan hydrogel containing fibroblast growth factor-2, Wound Repair Regen. 13 (2005) 390-397

[36] [36] K. Obara, M. Ishihara, T. Ishizuka, M. Fujita, Y. Ozeki, T. Maehara, et al., Photocrosslinkable chitosan hydrogel containing fibroblast growth factor-2 stimulates wound healing in healing-impaired $\mathrm{db} / \mathrm{db}$ mice, Biomaterials 24 (2003) 3437-3444

[37] [37] Tao Jiang, Roshan James, Sangamesh G. Kumbar, Cato T. Laurencin, Chitosan as a Biomaterial: Structure, Properties, and Applications in Tissue Engineering and Drug Delivery, Natural and Synthetic Biomedical Polymers, Chapter 5, 91-113

[38] [38] George Dan Mogosanu, Alexandru Mihai Grumezescu, Natural and synthetic polymers for wounds and burns dressing, International Journal of Pharmaceutics 463 (2014) 127- 136 